NORMAS. REVISTA DE ESTUDIOS LINGÜÍSTICOS HISPÁNICOS, N. o 3, 2013, PP. 161-181, ISSN: 2174-7245

(RECIBIDO: 2-09-2013. EVALUADO: 17-09-2013. ACEPTADO: 30-09-2013)

https://doi.org/10.7203/Normas.3.4677

\title{
HABLANTES DE HERENCIA DEL ESPAÑOL EN ESTADOS UNIDOS: IMPLICACIONES PARA EL ENTRENAMIENTO DE INSTRUCTORES EN EL NIVEL UNIVERSITARIO
}

\author{
SPEAKERS OF SPANISH AS A HERITAGE LANGUAGE IN THE UNITED STATES: \\ IMPLICATIONS FOR TEACHER TRAINING AT THE UNIVERSITY LEVEL
}

\author{
Lina María REZNICEK-PARRADO \\ Universidadde Nebraska-Lincoln*
}

\section{RESUMEN:}

Este estudio investiga las necesidades pedagógicas de los estudiantes de español como lengua de herencia (LH) en el nivel universitario. Basado en un análisis del perfil del hablante de herencia del español en el contexto de Estados Unidos y en un estudio del Departamento de Lenguas y Literaturas Modernas de la Universidad de Nebraska-Lincoln como caso ilustrativo, esta investigación busca explorar argumentos que apoyen la necesidad de proporcionar un entrenamiento a instructores de español del nivel universitario que responda a las necesidades pedagógicas específicas de los hablantes de herencia.

PALABRAS CLAVE: hablantes de español como lengua de herencia, entrenamiento pedagógico universitario, caso ilustrativo, Estados Unidos.

\section{ABSTRACT:}

This study researches the pedagogical needs of learners of Spanish as a Heritage Language (HL) at the university level. Based on an analysis of the Heritage Language student profile in the context of the United States and by using the University of Nebraska-Lincoln Modern Languages and Literatures Department as an illustrative case, this study seeks to explore arguments in favor of providing training for university-level instructors of Spanish that responds to the specific pedagogical needs of Heritage Language Learners.

KEYWORDS: Heritage speakers of Spanish, university teacher training, illustrative case, United States.

* Aunque el presente estudio se llevó a cabo en el Departamento de Lenguas y Literaturas Modernas de la Universidad de Nebraska-Lincoln, la autora es actualmente estudiante graduada del Departamento de Español y Portugués de la Universidad de California, Davis.

NORMAS. REVISTA DE ESTUDIOS LINGÜÍSTICOS HISPÁNICOS, NÚMERO 3 (AÑO 2013):

http://www.uv.es/normas

(ISSN 2174-7245) 


\section{INTRODUCCIÓN}

Este estudio demuestra, utilizando como caso ilustrativo el análisis de una encuesta administrada a instructores universitarios de español, la necesidad que sigue existiendo de concebir nuevos métodos de entrenamiento e instrucción que respondan a la diversidad del cuerpo estudiantil en este siglo XXI. Como extensión a la investigación actual, los resultados de este estudio exploran argumentos que apuntan a la necesidad de proporcionar un entrenamiento educativo para instructores de español que responda de manera apropiada a las necesidades pedagógicas específicas de los estudiantes de español como lengua de herencia. La relevancia de este estudio está reflejada no solo en el aumento de inscripciones de estudiantes que hablan español como lengua de herencia en clases de español al nivel universitario en Estados Unidos, sino también en la necesidad urgente de desarrollar prácticas metodológicas inclusivas al nivel de planeación, entrenamiento de instructores y métodos pedagógicos, las cuales respondan a las necesidades lingüísticas y de instrucción de los estudiantes universitarios que estudian el español como lengua de herencia.

\section{El ESPAÑOL EN Estados Unidos}

Debemos recalcar la importancia del español como lengua de uso tanto en las comunidades como en las instituciones educativas de Estados Unidos. Según información del censo del año 2010, más de la mitad del crecimiento total de la población en los Estados Unidos entre los años 2000 y 2010 fue gracias al aumento de la población hispánica (U.S. Census Bureau 2011 Census Brief). En el estado de Nebraska, por ejemplo, la población hispánica ha aumentado en un 77 \% desde el año 2000 (Pew Hispanic Center, 2010). No sería sorprendente, además, que los cambios demográficos que están ocurriendo en estados como Carolina del Norte, en el que la población hispánica creció en un $400 \%$ en el periodo de 1990 al 2000, continúen (U.S. Census, 2001). El crecimiento de las comunidades hispánicas en el país es sinónimo de una expansión proyectada del español, que convertirá a los Estados Unidos en los próximos años en el quinto país del mundo donde más se habla español (Lynch, 2003). Tal como afirma Roca (2000: 28):

Hoy en día la presencia hispánica pareciera estar en cualquier lado al que miremos, inclusive en áreas en donde menos nos lo esperaríamos, tal como pequeñas ciudades y pueblos en Carolina del Norte, Minnesota o Iowa. Los hispanos ya no estamos presentes únicamente en Nueva York, California, Texas o Florida.

Aunque la concentración más relevante de población hispánica sigue estando presente en nueve estados principalmente (California, Arizona, Nuevo México, Texas, Florida, Nueva York, Nueva Jersey, Colorado e Illinois), está demostrado que la movilidad de los hispanos repercute más allá y a través de la gran mayoría de los estados de la unión.

NORMAS. REVISTA DE ESTUDIOS LINGÜÍSTICOS HISPÁNICOS, NÚMERO 3 (AÑO 2013):

http://www.uv.es/normas

(ISSN 2174-7245) 
Si bien los cambios demográficos que muestran un crecimiento importante en la población hispánica al nivel nacional tienen repercusiones importantes dentro de las instituciones educativas, hay que notar que la presencia del español como materia de estudio en las instituciones de educación tiene una historia luenga e importante, relevante mucho antes de la ola de inmigración hispánica de los años 80.

En septiembre del 2003, la Asociación de Lenguas Modernas presentó los resultados de una encuesta llevada a cabo en el 2002 en la que participaron 2781 instituciones de educación superior de dos y cuatro años ${ }^{1}$. En ella, se demostró cuáles eran las lenguas que más se estudiaron según datos de admisión a partir de los años 60 hasta la primera parte del siglo XXI en el nivel universitario en Estados Unidos. Estos resultados se encuentran a continuación:

\begin{tabular}{|c|c|c|c|c|c|c|c|}
\hline \multicolumn{8}{|c|}{ Inscripciones } \\
\hline & 1960 & 1970 & 1980 & 1990 & 1995 & 1998 & 2002 \\
\hline Español & 178689 & 389150 & 379379 & 533944 & 606286 & 656590 & 746267 \\
\hline Francés & 228813 & 359313 & 248361 & 272472 & 205351 & 199064 & 201979 \\
\hline Alemán & 146116 & 202569 & 126910 & 133348 & 96263 & 89020 & 91100 \\
\hline Italiano & 11142 & 34244 & 34791 & 49699 & 43760 & 49287 & 63899 \\
\hline $\begin{array}{l}\text { Lenguaje Americano } \\
\text { de Señas }\end{array}$ & - & - & - & 1602 & 4304 & 11420 & 60781 \\
\hline Japonés & 1746 & 6620 & 11506 & 45717 & 44723 & 43141 & 52238 \\
\hline Chino & 1844 & 6238 & 11366 & 19490 & 26471 & 28456 & 34153 \\
\hline Ruso & 30570 & 36189 & 23987 & 44626 & 24729 & 23791 & 23921 \\
\hline Hebreo & 3834 & 16567 & 19429 & 12995 & 13127 & 15833 & 22802 \\
\hline Árabe & 541 & 1333 & 3466 & 3475 & 4444 & 5505 & 10584 \\
\hline Portugués & 1033 & 5065 & 4894 & 6211 & 6531 & 6926 & 8385 \\
\hline Coreano & 168 & 101 & 374 & 2286 & 3343 & 4479 & 5211 \\
\hline TOTAL & 604496 & 1057389 & 864463 & 1125865 & 1079332 & 1133512 & 1321320 \\
\hline
\end{tabular}

Figura 1. Número de inscripciones en los doce idiomas más estudiados (excluyendo el latín y el griego).

Fuente: Welles, 2004.

A partir de 1970 el español se convirtió en la lengua con mayor número de inscripciones a nivel nacional, superando al francés, sobre todo en el año 2002, en el que contó con 544288 más inscripciones que el segundo idioma más estudiado en las universidades de Estados Unidos. Según Welles, dentro de la lista de los quince idiomas más estudiados, el español siempre le ha llevado ventaja al segundo grupo, compuesto por el francés y el alemán (2004). Dice la autora, «el español es y siempre ha sido el idioma más enseñado en universidades desde 1970, y continúa representando más de la mitad (53.4 \%) de todas las inscripciones $[\ldots] \gg(2004: 12)$.

\footnotetext{
${ }^{1}$ Todas las instituciones universitarias invitadas a participar en esta encuesta respondieron, con la excepción de 12. Esto significa que el $99.6 \%$ de universidades proporcionaron datos, representando así la mayor tasa de respuesta en la historia de encuestas de la Asociación de Lenguas Modernas hasta el año 2002 (Welles, 2004).
}

NORMAS. REVISTA DE ESTUDIOS LINGÜÍSTICOS HISPÁNICOS, NÚMERO 3 (AÑO 2013):

http://www.uv.es/normas

(ISSN 2174-7245) 


\section{El HABLANTE DE HERENCIA DEL ESPAÑOL EN ESTADOS UNIDOS:}

La investigación que apunta hacia una definición de lengua o hablante de herencia es extensa y variada. Dada la complejidad del caso, sin embargo, la investigación se mantiene dividida, y el campo no ha podido llegar a un consenso sobre una definición global que describa quiénes son los hablantes de herencia. Aún así, en el caso de los hablantes de español en Estados Unidos, gracias a la relevancia de este grupo y como respuesta tanto a los cambios demográficos que se experimentan actualmente en las instituciones educativas como a la demanda de cursos de español, los educadores y académicos se siguen dando la tarea de continuar explorando, a través de una discusión académica, cómo definir mejor a los estudiantes de español como lengua de herencia. Presentaremos, en este caso, la definición del hablante de herencia del español en Estados Unidos según consideraciones enfocadas en la adquisición de una lengua de herencia y en las características lingüísticas de este tipo de hablante.

\subsection{La adquisición de una lengua de herencia}

La mayoría de hablantes bilingües de adquisición sucesiva son miembros de comunidades lingüísticas minoritarias que adquieren su lengua nativa (L1) en el hogar y/o en su comunidad inmediata, y su segunda lengua (L2) cuando ingresan a la escuela. Estos hablantes experimentan un cambio en la calidad, cantidad y función de la información que reciben en L1 en un momento en que su L1 puede que no haya sido completamente adquirida. Aunque la tendencia sería explicar la adquisición de una lengua de herencia (LH) como un proceso similar o idéntico a la adquisición sucesiva de una L2, Montrul (2012) aboga por la necesidad de ver a la lengua de herencia en términos más específicos para así considerarla como fenómeno único. La investigadora reitera que la única manera de entender el proceso de adquisición de una lengua de herencia es teniendo en cuenta no solo el orden de adquisición de tanto la L1 vs. la L2, sino que es esencial tomar en cuenta las dimensiones funcionales de las lenguas que habla el individuo (lengua principal vs. lengua secundaria) al igual que los factores sociopolíticos (lengua minoritaria $v s$. lengua mayoritaria) que forman parte de este proceso de adquisición (2012).

Así, la adquisición de una lengua de herencia debe ser concebida como proceso, pues en el momento en que la L1 se convierte en una lengua minoritaria para el individuo, existe un cambio importante en la dimensión funcional de los dos idiomas hablados por el individuo mientras crece y mientras la L1, siendo la lengua principal, se convierte muy pronto en la lengua de uso secundario. Este cambio funcional, por su parte, afecta la competencia tanto lingüística como comunicativa de la L1 en el individuo (Montrul, 2012). 


\section{L1 $=$ Heritage Language \\ L2 = English (in the US)}

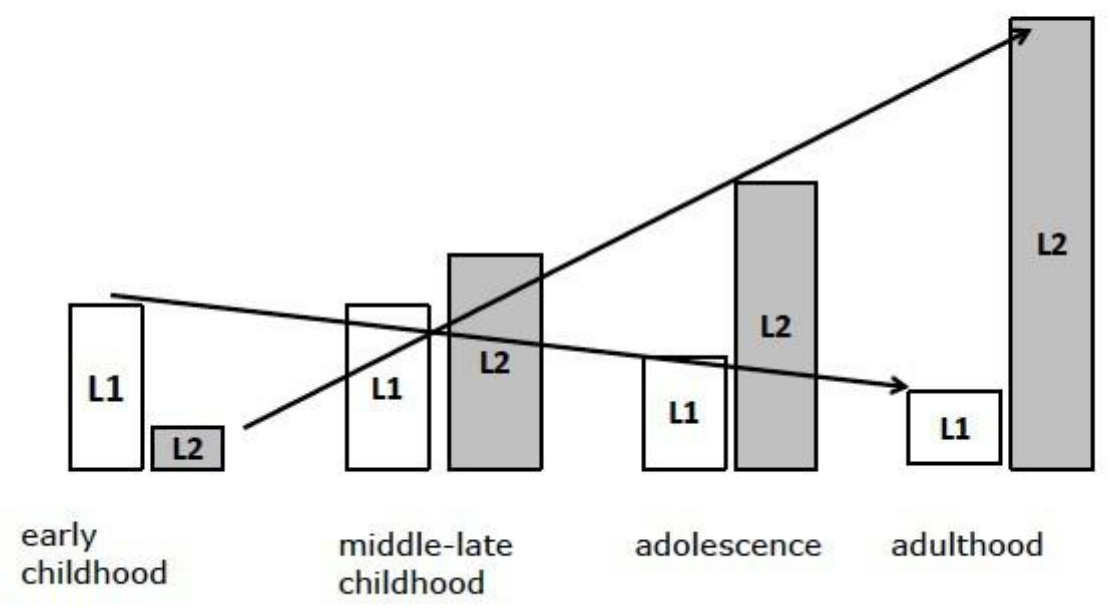

Figura 2. Desarrollo funcional típico de un hablante de herencia (L1) en un contexto de lengua mayoritaria. Fuente: Montrul, 2012.

Mucha de la investigación de los últimos años ha intentado explicar el porqué de los patrones linguiísticos que se observan en la producción de hablantes de lenguas heredadas. Aunque la investigación que intenta responder a esta pregunta sigue inconclusa, lo que sí encuentra general aceptación es que la adquisición de lenguas de herencia tiene características tanto de la adquisición de una lengua nativa (L1) como de la adquisición de una lengua segunda (L2), haciendo que la lengua de herencia resulte siendo un caso híbrido, producto de la combinación de estos dos procesos de adquisición.

\subsection{Características lingüísticas de los hablantes de herencia del español}

Guiados por el trabajo de Guadalupe Valdés (2001), una de las investigadoras más reconocidas dentro de la investigación de pedagogía de lenguas de herencia, los instructores de lenguas utilizan el término «lengua de herencia» para referirse a la lengua del estudiante «que creció en un hogar donde se habla una lengua diferente al inglés, que habla o al menos entiende tal lengua, y que es hasta cierto nivel bilingüe en tal lengua y en inglés» (Valdés 2000a, 2000b, citado en Valdés, 2001: 2).

Bajo el contexto de esta definición, Valdés (2001) reitera la importancia de reconocer que el término «bilingüe» no implica el uso de las dos lenguas a un nivel superior a tal punto que el individuo pueda llevar a cabo todo lo que hace en una lengua de la misma 
manera en la otra lengua, ni que pueda pasar inadvertido para un hablante monolingüe de cualquiera de las dos lenguas que habla. Quienes conceptualizan al estudiante bilingüe como alguien que demuestra un equilibrio idealizado y perfectamente equiparable entre las lenguas que habla están imaginando una figura mítica, y por ende, muy poco común de lo que implica en realidad el ser bilingüe.

El bilingüismo se describe mejor como una condición, o un proceso dinámico que está en constante movimiento. Esto explica por qué los estudiantes que hablan español como lengua de herencia en Estados Unidos presentan perfiles altamente variados. En unos casos y de acuerdo a su situación y experiencia personal, un estudiante puede demostrar habilidades mínimas en español, entender únicamente la lengua de uso cotidiano y usarla en contextos sociales limitados. De igual manera, este mismo grupo de estudiantes puede incluir individuos que manejan la lengua a tal nivel que se acercan al perfil lingüístico de un hablante nativo, tanto en términos de lo oral como de lo escrito.

Potowski $(2005,2008)$ explica que las principales características linguiísticas del español del hablante de herencia en el contexto norteamericano se definen a través de tres componentes principalmente: la interacción entre las formas prestigiosas versus las estigmatizadas; la alternancia de códigos; y la influencia del inglés, la cual está determinada por el grado de contacto del español con el inglés y por la necesidad de utilizar los dos idiomas de manera creativa en la vida cotidiana. Muchos investigadores se refieren a estos últimos dos componentes a través de la etiqueta «Spanglish» (Stavans, 2003, citado en Potowski, 2005).

Dada la diversidad del perfil demográfico y socioeconómico de este grupo, los hablantes de español como lengua de herencia no siempre están expuestos a la lengua estándar en su proceso de escolarización. La exposición al español que reciben puede limitarse a una variedad regional que contiene formas estigmatizadas (Potowski, 2008). Si este hablante no tiene la oportunidad ni el privilegio de recibir escolarización avanzada en español de manera tal que desarrolle los registros necesarios para la adquisición completa de la lengua, o si se trata de un hablante de una variedad rural con necesidad de desempeñarse en contextos cotidianos únicamente, las formas estigmatizadas pueden ser las únicas que produce. Sin embargo, Potwoski recuerda que muchas de las formas del español utilizadas por algunos grupos minoritarios en Estados Unidos y que ahora son estigmatizadas, como por ejemplo yo truje (yo traje), asina (asî), naiden (nadie), haiga (haya), entre otros, eran parte del español del pasado y hoy son clasificados como arcaísmos conservados del español de los descendientes de quienes colonizaron en el siglo XVI lo que es hoy en día el suroeste de Estados Unidos (2008:233). Así, «el uso de tales formas, igual que otras que no constituyen arcaísmos, merecen el respeto de los maestros dado que en el campo de la lingüística todas las variedades de un idioma se consideran igualmente valiosas» (Potowski, 2005: 22).

NORMAS. REVISTA DE ESTUDIOS LINGÜÍSTICOS HISPÁNICOS, NÚMERO 3 (AÑO 2013):

http://www.uv.es/normas

(ISSN 2174-7245) 
De igual manera, muchos de los hablantes del español como lengua de herencia hacen uso de las estructuras y vocabulario del inglés con el fin de expresar mejor su experiencia cotidiana. Potowski (2005) señala que son tres los fenómenos lingüísticos más comunes y presentes en el español de Estados Unidos como producto de la influencia del inglés: los préstamos léxicos, las extensiones semánticas y los calcos del inglés. Todos estos fenómenos, incluyendo la alternancia de códigos, son manifestaciones de la categoría que se conoce como «Spanglish».

La consideración del perfil lingüístico del hablante de herencia del español en Estados Unidos demuestra la importancia de la experiencia lingüística única de este tipo de hablante, además de las repercusiones sociopolíticas y de identidad que implica la presencia del español como lengua de herencia en Estados Unidos.

\section{Un CASO ILUSTRATIVO DEL MidWEST}

Figura 3. Ubicación geográfica del estado de

Aunque Nebraska, a diferencia de estados como Florida, California, o Texas, no ha sobresalido tradicionalmente como uno de los estados de mayor población hispánica en los Estados Unidos, es relevante resaltar los cambios recientes que han afectado la composición de este estado del Midwest americano. En el 2000, la población total de hispanos en un estado de más o menos 1700000 personas era de 94 000, mientras que en el 2010 se reportó una población hispana total de 168 000, teniendo en cuenta que la población estatal cerró en 1800000 personas (Pew Hispanic Center, 2010b). Así, la población hispana a nivel estatal creció en un $77 \%$ en un periodo de apenas diez años.

Los censos anteriores al 2010 demuestran, de igual manera, que existen condados de alta población en el estado de Nebraska que ya habían tenido un aumento importante en la población hispánica. Interesante también es el crecimiento de esta población entre el 2000 y el 2010 en algunos condados de poca población: en el condado de Holt (población total de 10435 en el año 2010) la población hispánica creció en un $272 \%$; en el condado de Webster (población total de 3812 en el año 2010) el crecimiento fue de $505 \%$; y en el condado de Boyd (población total de 2099 en el año 2010) el censo reporta un crecimiento de 1550 \% (Pew Hispanic Center, 2013a). Aunque estas comunidades no representan las áreas de mayor concentración de población en el estado, es importante considerar que la presencia de la población hispánica abarca tanto las zonas rurales como las metropolitanas del estado. 
Uno de los rasgos más significativos de la composición hispánica en el estado de Nebraska es la edad. El perfil demográfico de los hispanos en Nebraska muestra que de los 168000 habitantes, tan solo el $34 \%$ nacieron en el extranjero. Esto significa que más de la mitad de los hispanos en Nebraska (66 \% para ser exactos) son nacidos en los Estados Unidos, implicando la posibilidad de que, si no la mayoría, muchos de sus integrantes podrían ser menores de edad o adultos jóvenes. Quizá mucho más relevante aún es el hecho que el $15 \%$ de todos los niños y adolescentes inscritos en escuelas de grados kínder hasta el doceavo son latinos, posicionando a Nebraska en el decimoctavo puesto a nivel nacional en términos de número de estudiantes hispanos dentro del sistema escolar preuniversitario (Pew Hispanic Center, $2013 b)$.

Los datos demográficos presentados por el Pew Hispanic Center implican que la población hispánica del estado de Nebraska es joven (la edad media de los hispanos en Nebraska es de 22 años de edad). De igual manera, basado en datos del American Community Survey del 2010, el Pew Hispanic Center también reporta que de los 168000 hispanos en este estado, el $66 \%$ de aquellos mayores de 5 años hablan español en casa (Pew Hispanic Center, 2013b).

\subsection{La Universidad de Nebraska-Lincoln: cambios demográficos}

Un análisis del cambio demográfico dentro de la principal institución de educación pública superior del estado de Nebraska sugiere la necesidad no solo de considerar el impacto actual del crecimiento en el número de estudiantes hispanos, sino también en la situación del departamento que se encarga de enseñar el idioma que muchos de ellos hablan en casa. Si la mayoría de los habitantes hispanos en Nebraska son adultos jóvenes y muchos de ellos están en edad universitaria, además de que $15 \%$ de ellos cursan actualmente grados preuniversitarios, la proyección apuntaría a que muchos de ellos podrían optar por asistir a la Universidad de Nebraska-Lincoln ${ }^{2}$. No sería descabellado tampoco proyectar que muchos de estos estudiantes podrían tener interés en tomar clases de español.

En el 2011, la Universidad de Nebraska-Lincoln (UNL por sus siglas en inglés) tenía una población estudiantil de aproximadamente 25000. Según información reportada por el Centro Nacional de Estadísticas de la Educación, en el 2008 el $2.7 \%$ de los estudiantes inscritos en UNL eran hispanos, mientras que en el año escolar del 2011-2012, los estudiantes hispanos representaron el $4.2 \%$ de todas las inscripciones. En el 2011 la UNL reportó el mayor número de estudiantes hispanos de pregrado inscritos en la historia de esta institución: 817 estudiantes de pregrado, incluyendo 225 estudiantes de primer año (más de una tercera parte del número total de estudiantes hispanos inscritos el año previo) (Holtmeyer, 2011).

${ }^{2}$ Un estudio reciente conducido por el Pew Hispanic Center demuestra que a nivel nacional, las inscripciones universitarias de estudiantes hispanos aumentó en un $24 \%$ entre el año 2009 y 2010 (Holtmeyer, 2011).

NORMAS. REVISTA DE ESTUDIOS LINGÜÍSTICOS HISPÁNICOS, NÚMERO 3 (AÑO 2013):

http://www.uv.es/normas

(ISSN 2174-7245) 


\subsection{El Departamento de Lenguas y Literaturas Modernas: la sección de español}

Durante el semestre en el que se llevó a cabo el presente estudio (primavera del 2012), la sección de español ofreció aproximadamente unos 70 cursos de español, incluyendo desde ocho secciones del primer nivel hasta cursos de literatura y gramática avanzada, entre otros. En este semestre todos los cursos de español ofrecidos fueron cursos de lengua extranjera (LE); no se ofreció ningún curso específicamente para estudiantes que hablan español como lengua de herencia (LH). En términos de inscripciones, la sección de español contó con 1485 estudiantes inscritos en clases de pregrado y de posgrado (58\% de las inscripciones de todo el departamento). Dentro de este grupo, 120 estudiantes declararon el español como su especialización y 458 como subespecialización. De igual manera, 19 estudiantes graduados formaron parte de la sección de español durante este semestre, cuatro de ellos a nivel maestría y 15 a nivel doctorado. La mayoría de instructores en este departamento hablan una variedad peninsular del español (el $63 \%$ de los participantes) mientras que el $32 \%$ hablan una variedad latinoamericana del español. Ningún instructor habla una variedad del español en Estados Unidos.

\section{3. «La enseñanza del español al nivel universitario»: una encuesta}

Durante la primavera del 2012 y como parte del presente estudio, todos los instructores, incluyendo a profesores, adjuntos y asistentes de la sección de español del Departamento de Lenguas y Literaturas Modernas en la Universidad de Nebraska-Lincoln fueron contactados por vía electrónica para ser invitados a participar en una encuesta acerca de la enseñanza del español en el nivel universitario ${ }^{1}$. Los participantes podían tener acceso a esta encuesta, administrada a través de <www.surveymonkey.com>, a través de un enlace incluido dentro de la correspondencia electrónica. El presente estudio no se presentó a los participantes como un estudio sobre los hablantes de herencia del español específicamente, teniendo en cuenta que el objetivo de la encuesta era el de incitar las opiniones de los instructores sobre la enseñanza del español en el nivel universitario como tema de implicación general. La intención de la encuesta era analizar las opiniones y perspectivas de los instructores desde un punto de vista general, personal, poco técnico, y basado única y exclusivamente en la experiencia profesional existente del participante.

Al cabo de aproximadamente tres meses, 15 asistentes, 9 adjuntos y 6 profesores respondieron la encuesta, representando el $85 \%$ de todos los instructores que enseñaron una clase de español ese semestre.

\subsubsection{Percepción: ¿Quién es el hablante de herencia que estudia español?}

Con el fin de conocer las percepciones de los instructores sobre quién es el hablante de herencia, primero se le preguntó al instructor si durante su experiencia profesional en UNL había tenido «hablantes nativos de español».

\footnotetext{
${ }^{1}$ Tanto el presente estudio como el diseño de la encuesta fueron aprobados por parte del Institutional Review Board (IRB por sus siglas en inglés) el 2 de abril del 2012.
}

NORMAS. REVISTA DE ESTUdIOS LINGÜÍSTICOS HISPÁNICOS, NÚMERO 3 (AÑO 2013):

http://www.uv.es/normas

(ISSN 2174-7245) 
Los resultados a esta pregunta se muestran a continuación:

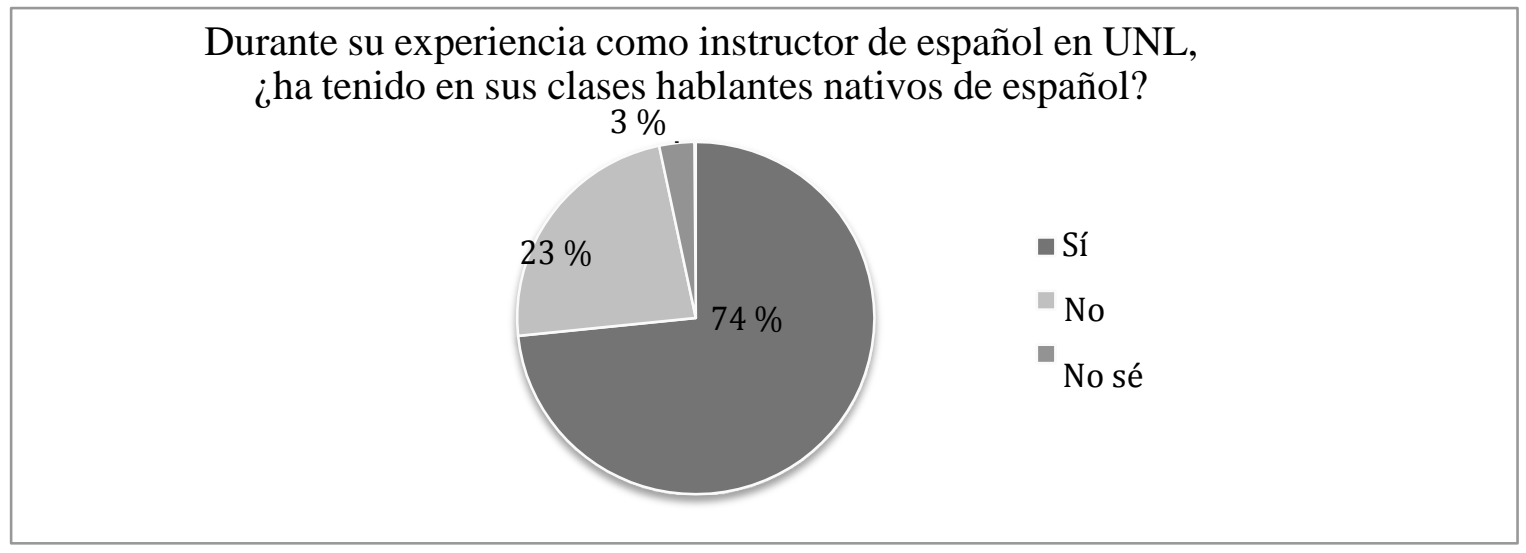

Figura 4. Resultados a la pregunta número 8: «Durante su experiencia como instructor de español en UNL, ¿ha tenido en sus clases hablantes nativos del español»?

Dos preguntas después y con el fin de distinguir entre estudiantes internacionales de países hispanohablantes (aquellos que llegan a la institución como estudiantes de intercambio o con el objetivo único de cursar una carrera profesional) y estudiantes que hablan el español como lengua de herencia, se invitó al instructor a pensar si tenía estudiantes «que se criaron en Estados Unidos y que aprendieron español en casa»:

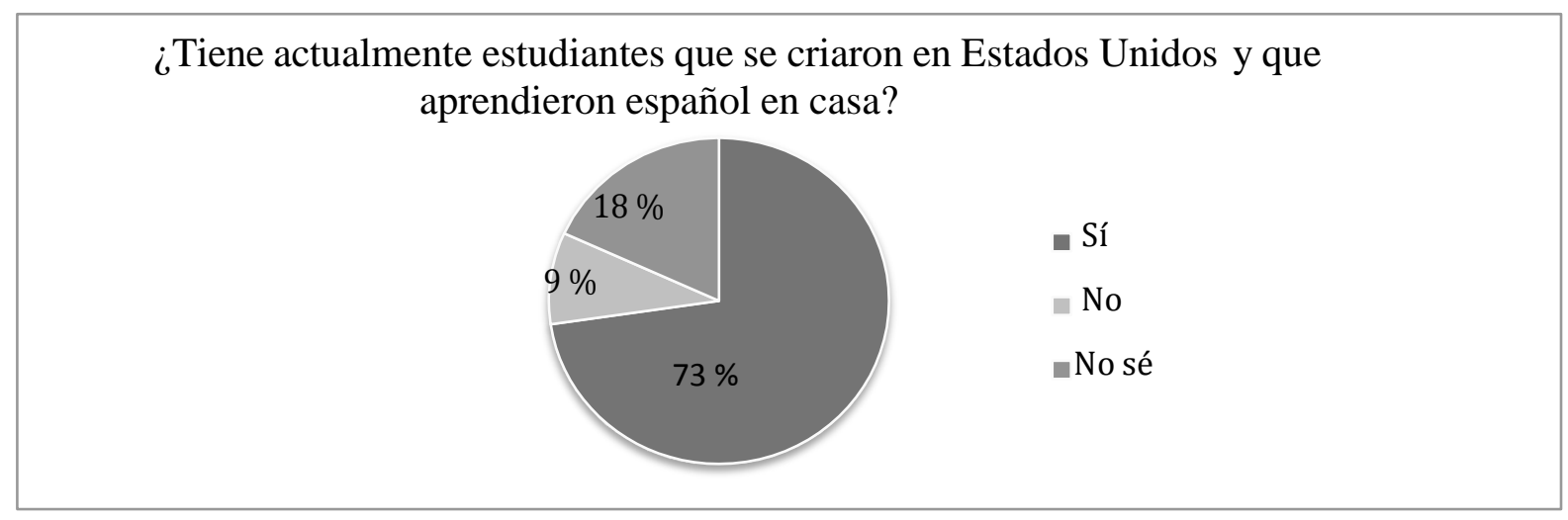

Figura 5. Resultados a la pregunta número 10: «¿Tiene actualmente estudiantes que se criaron en Estados Unidos y que aprendieron español en casa?»

NORMAS. REVISTA DE ESTUDIOS LINGÜÍSTICOS HISPÁNICOS, NÚMERO 3 (AÑO 2013):

http://www.uv.es/normas 
Al comparar estos dos gráficos, y al únicamente cambiar «hablantes nativos» por «hablantes nativos criados en Estados Unidos que aprendieron español en casa», es interesante que el porcentaje de la categoría «No sé» haya aumentado de un $3 \%$ a un $18 \%$. Esto podría ser una indicación importante de que el instructor que responde a la encuesta no está seguro si a) existe una diferencia entre el estudiante «hablante nativo del español» y el estudiante «criado en Estados Unidos que aprendió español en casa»; o b) que el instructor está definiendo indiscriminadamente al estudiante «criado en Estados Unidos que aprendió español en casa». Así pues, no pareciera existir un consenso entre los participantes en términos de una definición clara y consistente sobre quién es el hablante de herencia que estudia español.

La hipótesis anterior parece reforzarse en el momento de analizar los resultados de la pregunta número nueve: «Al principio del semestre, ¿cómo sabe que tiene hablantes nativos del español en su(s) clase(s)?». Los temas que surgen de los resultados a esta pregunta-habilidades orales y/o de escritura, experiencia fuera del salón de clase, y nombre y apellido del estudiante, apuntan a que no existe un consenso, y que la diferencia entre hablante nativo y hablante de herencia era poco clara para este grupo de instructores:

\begin{tabular}{|c|c|}
\hline Tema & Respuesta \\
\hline \multirow{13}{*}{$\begin{array}{l}\text { Perfil hablante nativo } \\
\text { Habilidades orales y/o de escritura }\end{array}$} & La fluidez con la que escriben. \\
\hline & Porque hablan español de manera fluida. \\
\hline & Pero el problema de ellos es la gramática, la escritura. \\
\hline & El acento hablado. \\
\hline & Su español es excelente. \\
\hline & $\begin{array}{l}\text { Los hablantes nativos se distinguen por su nivel del habla } \\
\text { de los demás estudiantes. }\end{array}$ \\
\hline & Se comunican oralmente sin problemas. \\
\hline & No tienen bases para la escritura. \\
\hline & La forma de hablar, si pronuncian bien o no. \\
\hline & Conocen aspectos gramaticales no enseñados en clase. \\
\hline & Escritura semejante a la forma cotidiana de hablar. \\
\hline & Mas seguridad a la hora de expresarse en español. \\
\hline & $\begin{array}{l}\text { Pueden entender todo y hablar ya, usando gramática avanzada y } \\
\text { expresiones que sólo un nativo sabría/podría usar/decir. }\end{array}$ \\
\hline \multirow{7}{*}{$\begin{array}{l}\text { Perfil hablante nativo } \\
\text { Experiencia por fuera del salón de clase }\end{array}$} & Su experiencia con el español. \\
\hline & $\begin{array}{l}\text { Algunos de los estudiantes que tuve me comentaron que en la } \\
\text { casa sólo hablan español. }\end{array}$ \\
\hline & $\begin{array}{l}\text { Por medio de algunas tareas donde llegamos a conocer el } \\
\text { background del estudiante. }\end{array}$ \\
\hline & Les pregunto que si se habla español en su familia. \\
\hline & Ellos me hablaron de su herencia y de su familia. \\
\hline & Si alguien habla español en casa. \\
\hline & Aprendido desde otro lugar o comunidad de habla hispana. \\
\hline \multirow{5}{*}{$\begin{array}{l}\text { Perfil hablante nativo } \\
\text { Nombre y apellido }\end{array}$} & El nombre. \\
\hline & $\begin{array}{l}\text { Los apellidos, me da un buen indicio si es hablante de herencia } \\
\text { o no. }\end{array}$ \\
\hline & Por los nombres de los estudiantes. \\
\hline & Su nombre. \\
\hline & Si reconozco los apellidos como hispanos. \\
\hline
\end{tabular}

Figura 6. Resultados a la pregunta 9: «Al principio del semestre, ¿cómo sabe que tiene hablantes nativos del español en su(s) clase(s)?». 
El $23 \%$ de las respuestas pueden agruparse bajo el tema experiencia por fuera del salón de clase. Dentro de este tema, parece que sí existe una noción entre algunos instructores de la dimensión sociopolítica y personal del hablante de herencia en términos de su relación con el español. Respuestas como «me hablaron de su herencia», «se habla español en casa», «su experiencia con el español» apuntan a que el estudiante en cuestión está siendo considerado como hablante de herencia basado en su realidad compleja con el idioma por fuera del salón de clase. Sin embargo, el $43 \%$ de las respuestas que agrupamos aquí bajo el tema habilidades orales y/o escritas, presentan varias oposiciones. Aunque pareciera haber una noción de que el estudiante de herencia presenta habilidades orales avanzadas, parece no haber consenso en cuanto a sus habilidades de escritura. Se incluyeron respuestas para definir al hablante de herencia que determinaban una «fluidez con la que escriben» pero también respuestas como «el problema de ellos es la gramática, la escritura» y «no tienen bases para la escritura». De igual manera, las respuestas «conocen aspectos gramaticales no enseñados en clase» y «pueden entender todo y hablar ya, usando gramática avanzada (...)» no concuerdan con la realidad lingüística del hablante de herencia. Quizá lo más problemático al momento de analizar las percepciones del perfil del hablante de herencia es que casi el $20 \%$ de los instructores que participaron en este estudio se basaron principalmente en el nombre o apellido del estudiante para determinar si era hablante de herencia o no. Tomando en cuenta la realidad demográfica del cuerpo estudiantil, es esencial saber que un nombre hispánico no implica necesariamente que un individuo hable español, y por otra parte, que muchos hispanos no tienen un apellido en español.

\subsubsection{Percepción: ¿Por qué toman clases de español los hablantes de herencia?}

Uno de los temas principales a los cuales se quería acercar la presente encuesta era las percepciones de los participantes sobre las razones por las cuales los hablantes de herencia, definidos como « hablantes nativos del español que se criaron en Estados Unidos », toman clases de español en el nivel universitario. Las respuestas a esta pregunta pueden agruparse bajo tres temas principales: mejorar habilidades de lectoescritura, reconectarse con su cultura/herencia, y obtener créditos fáciles. El tema más recurrente, apareciendo casi en el 50 $\%$ de las respuestas, fue el de obtener créditos fáciles o cumplir con un requisito académico. Las diferentes expresiones de este tema se presentan a continuación: 


\begin{tabular}{|c|c|}
\hline Tema & Respuesta \\
\hline \multirow{14}{*}{$\begin{array}{l}\text { Razones para tomar español } \\
\text { Créditos fáciles }\end{array}$} & Porque piensan que va a ser fácil. \\
\hline & Para cumplir con el language requirement. \\
\hline & $\begin{array}{l}\text { Porque piensan que pueden obtener los créditos sin } \\
\text { demasiado esfuerzo. }\end{array}$ \\
\hline & $\begin{array}{l}\text { Creo que se registran para conseguir fácilmente } \\
\text { créditos. }\end{array}$ \\
\hline & $\begin{array}{l}\text { En algunos casos lo hacen porque creen que será } \\
\text { una clase fácil para ellos. }\end{array}$ \\
\hline & $\begin{array}{l}\text { Para acumular créditos y sacar ventaja para su GPA } \\
\text { al obtener buenas notas. }\end{array}$ \\
\hline & Es fácil. \\
\hline & $\begin{array}{l}\text { Para obtener un título académico (especialización o } \\
\text { subespecialización). }\end{array}$ \\
\hline & Porque les resultan fáciles los cursos básicos. \\
\hline & En general, para obtener créditos fáciles. \\
\hline & para tener una calificación buena de manera fácil. \\
\hline & $\begin{array}{l}\text { Hay un porcentaje pequeño que piensan que, como } \\
\text { hablan el español, todo va a ser un camino de rosas. }\end{array}$ \\
\hline & Por creer en su supuesta facilidad. \\
\hline & $\begin{array}{l}\text { Primero pensaba que tomaron la clase para recibir } 5 \\
\text { créditos de } \mathrm{A}+\text {. }\end{array}$ \\
\hline
\end{tabular}

Figura 7. Resultados a la pregunta 13: «En su opinión, ¿por qué toman clases de español en la Universidad los estudiantes que son hablantes nativos del español y que se criaron en Estados Unidos?».

Aunque es necesario llevar a cabo un estudio completo sobre las verdaderas razones por las que los hablantes de herencia toman clases de español en este departamento y otros de composición similar, varios estudios anteriores han examinado las razones por las que muchos hablantes de herencia se inscriben en cursos universitarios para estudiar su lengua de familia. Carreira y Kagan (2011), por ejemplo, condujeron una encuesta a nivel nacional para estudiar las razones por las cuales un grupo de estudiantes universitarios que eran hablantes de herencia del español, árabe, armenio, cantonés, vietnamita, entre otros, tomaban clases en sus respectivas lenguas de herencia.

Las investigadoras encontraron que las prioridades más importantes de los hablantes de herencia que tomaban cursos de lengua en la universidad eran, de mayor a menor relevancia:

1) aprender sobre sus raíces culturales y lingüísticas;

2) mejorar su comunicación con familiares y amigos en los Estados Unidos;

3) como objetivo puramente pragmático, para llenar un requisito académico (48). 
Aunque la noción de créditos fáciles también está presente en el estudio de Carreira y Kagan, las investigadoras concluyen, dada la poca relevancia de esta última prioridad, que en resumen, «el perfil del típico estudiante hablante de herencia según los datos fue el de un estudiante que tiene, sobre todo, sentimientos y experiencias positivas en cuanto a su lengua de herencia y para quienes las razones para estudiar esta lengua son sobre todo personales y no académicas (...)» (Carreira y Kagan, 2011: 48). De igual manera, al analizar resultados específicamente tomando en cuenta a los estudiantes de español como lengua de herencia, las investigadoras demostraron que, al igual que los hablantes de herencia del mandarín y el cantonés, los hablantes de herencia del español fueron los únicos para quienes los objetivos profesionales resultaron más importantes que los objetivos personales. Específicamente, el $71 \%$ de los participantes clasificados como hablantes de herencia del español en ese estudio dijeron estudiar su lengua de herencia teniendo en cuenta su carrera profesional (Carreira y Kagan, 2011: 51). Como puede verse, los resultados de Carreira y Kagan contrastan de manera importante con las percepciones de los instructores que participaron en el presente estudio.

\subsubsection{Percepción: ¿Por qué no toman clases de español los hablantes de herencia?}

Otro de los temas principales a los que se quería acercar la encuesta era las razones percibidas por los instructores de por qué los estudiantes «nativos del español que se criaron en Estados Unidos» podrían preferir no inscribirse en las clases de español ofrecidas en el departamento. En respuesta a esta pregunta surgieron dos temas principales: dificultades estructurales del curso y sentimientos negativos hacia el español. 


\section{Tema}

Razones para no tomar español

Dificultades estructurales del curso

Razones para no tomar español

Sentimientos negativos por el español

\section{Respuesta}

La clase está diseñada para los que están aprendiendo español, y no tenemos un componente específico que les ayudaría a los hablantes de herencia. Por ejemplo, es muy difícil intentar a enseñar cosas que son beneficiosas para hablantes de herencia (ortografía, acentos, puntuación, tono, registro, etc.) cuando la mayoría de la clase no puede ni componer bien una oración básica. La mayoría de la clase se nos pasa repasando la gramática básica que un hablante de herencia ya domina bastante bien. Por ende, se aburren y no les gustan nuestras clases.

Nuestro programa tiene sus limitaciones, no todos están interesados en la cultura, la lengua y la literatura. Sería bueno tener más opciones para este tipo de estudiantes.

Es difícil ubicarse en un nivel, así que algunos materiales pueden parecer muy fáciles y otros muy difíciles

It's possible that some people may feel embarrassed about their level of proficiency in their native language.

They may also feel undue embarrassment if their particular dialect is not considered is not seen as having the "prestige" of others.

No están interesados en mejorar su español.

Que el español no es un idioma considerado "culto» o «elegante» en Estados Unidos como el francés.

El español es la lengua de casa y nada más.

No ven practicidad y uso en el estudio formal de la lengua.

Inseguridad en la lengua.

Falta de interés.

No querer formar parte de la identidad latina por la discriminación que existe.

Consideran que no es importante para su vida profesional y personal.

Podrían sentir que son irrelevantes para ellos. Piensan que lo que saben es suficiente.

Pensar que ya saben español.

Creen suficiente su conocimiento del español y no vean necesario tomar clases de gramática, estilo, lectura, etc.

El estatus académico de todo lo relativo al español es bajo.

También deseo de integrarse en la población blanca no hispana y borrar toda traza de origen hispano.

Vergüenza por no poder hablar bien. 


\begin{tabular}{|ll|}
\hline & No ver el beneficio. \\
\hline $\begin{array}{l}\text { Miedo de sentirse o muy aburrido o muy } \\
\text { presionado. }\end{array}$ & $\begin{array}{l}\text { Les parece innecesario tomar clases de una lengua } \\
\text { que creen conocer y manejar bien. }\end{array}$ \\
\hline $\begin{array}{l}\text { Ignorancia y desprecio hacia la cultura y lengua } \\
\text { española por los estereotipos fuertemente marcados } \\
\text { por la sociedad estadounidense para relacionar la } \\
\text { lengua española con la del pobre y el migrante. }\end{array}$ \\
\hline $\begin{array}{l}\text { Falta de interés en su desarrollo lingüístico en } \\
\text { español o por temor a mostrar el desconocimiento } \\
\text { de una lengua que socialmente se les atribuye como } \\
\text { propia (...) }\end{array}$ \\
\hline \begin{tabular}{l} 
No quieren pasar tiempo en clase. \\
\hline No se dan cuenta de la importancia del idioma.
\end{tabular} \\
\hline $\begin{array}{l}\text { Ellos creen que hablar es suficiente }(. . .) \text { Tomar un } \\
\text { curso de español les va a quitar algo de tiempo, } \\
\text { entonces, prefieren concentrarse más en su área de } \\
\text { estudio. }\end{array}$ \\
\hline $\begin{array}{l}\text { (...) desconexión entre la vida de casa y la vida de } \\
\text { universidad (...) Puede que algunos hablantes } \\
\text { nativos prefieran que su bilingüismo sea aspecto de } \\
\text { su vida casera. }\end{array}$ \\
\hline $\begin{array}{l}\text { Porque creen que saben todo lo que es necesario } \\
\text { para obtener un buen trabajo sin cursos en español. }\end{array}$ \\
\hline Quieren avanzar directamente a su especialización. \\
\hline $\begin{array}{l}\text { Creo que sus razones son personales y relacionadas } \\
\text { con obligaciones que tienen fuera de la Universidad. }\end{array}$ \\
\hline Que ya hablan español. \\
\hline $\begin{array}{l}\text { Quizás sea porque ya saben hablar y prefieren } \\
\text { seguir otro camino que la enseñanza del idioma. }\end{array}$ \\
\hline
\end{tabular}

Figura 8. Respuesta a la pregunta 19: «En su opinión, ¿qué motivas podrían tener los hablantes nativos del español que se criaron en Estados Unidos para preferir no tomar nuestras clases?».

Según la tabla anterior, únicamente el $10 \%$ de los encuestados consideraron las dificultades estructurales de los cursos de segunda lengua ofrecidos por el departamento como posible razón para que los hablantes de herencia decidan no tomar cursos de español. En otras palabras, únicamente este porcentaje percibía como deficiencia del departamento el que un hablante de herencia pudiera no querer tomar clases de español. El $90 \%$ de las respuestas proporcionadas por los encuestados mencionó que los posibles motivos para no tomar clases de español estribaban principalmente en el estudiante. Entre estos motivos se encontraban posibles sentimientos negativos incluyendo vergüenza, desprecio, inseguridad, falta de practicidad, falta de importancia y falta de interés. Para la gran mayoría de los encuestados en este estudio, las razones por las que los hablantes de herencia no tomaban clases de español eran razones externas al departamento, y por ende, razones que se encontraban más allá de su control como instructores.

NORMAS. REVISTA DE ESTUDIOS LINGÜÍSTICOS HISPÁNICOS, NÚMERO 3 (AÑO 2013):

http://www.uv.es/normas 
Estos resultados contrastan también con los resultados de Carreira y Kagan (2011), quienes demostraron que el estudiante de herencia presenta actitudes muy positivas sobre su lengua de herencia. Entre las actitudes sobre su lengua de herencia de mayor porcentaje proporcionadas por los estudiantes de herencia universitarios encuestados por Carreira y Polinsky estaban las siguientes: es una habilidad valiosa-89 \%; me parece que es útil-86\%; es una parte importante de quién soy-85\%; es una habilidad necesaria-71\%; me ha ayudado a hacer más amigos- $52 \%$ y ha hecho que disfrute más la escuela - 46 \% (Carreira y Kagan, 2011: 47).

Aunque el número de participantes hace que las conclusiones de este estudio no sean generalizables, estos resultados y su oposición a estudios similares recientes sugieren la necesidad de estudiar las percepciones de los instructores de español para entender cómo mejor responder a las necesidades pedagógicas de los hablantes de herencia que se inscriben en cursos de español a nivel universitario.

\section{CONCLUSIÓN E IMPLICACIONES}

Las necesidades pedagógicas de los hablantes de herencia de español de nivel universitario implican una consideración lingüística de adquisición de lenguas que tome en cuenta tanto teorías establecidas de adquisición de una lengua materna (L1) al igual que de una segunda lengua (L2), considerando que muchos de los factores de estos dos procesos son relevantes en el momento de describir la adquisición de una lengua de herencia (LH), aunque no es en todos. Así, estos tres procesos de adquisición deben ser analizados en términos de categorías diferentes, pero categorías que tienen mucho para aportar entre sí.

Los instructores de español de nivel universitario que tienen estudiantes que hablan el español como lengua de herencia deben recibir un entrenamiento apropiado, el cual aporte información sobre las diferencias en necesidades pedagógicas entre este grupo de estudiantes y los estudiantes de lengua extranjera. Schwartz (2001) sugiere que además de la preparación en español a la que los instructores probablemente ya han estado expuestos, esta preparación profesional debe incluir también conceptos provenientes de una diversidad de disciplinas, tal como son la lingüística, la psicolingüística, la sociolingüística, la lingüística aplicada y la psicología. Los instructores deben conocer los principales procesos linguiísticos de adquisición de lenguas L1, L2 y LH, incluyendo la adquisición de una variedad estándar por parte de hablantes de variedades estigmatizadas.

NORMAS. REVISTA DE ESTUDIOS LINGÜÍSTICOS HISPÁNICOS, NÚMERO 3 (AÑO 2013):

http://www.uv.es/normas

(ISSN 2174-7245) 
De igual manera, los instructores deben recibir un entrenamiento que les ayude a ser conscientes de la manera en que factores como la edad, el contexto sociopolítico y la identidad afectan a la adquisición del español como lengua de herencia, y así desarrollar una actitud sensible en el momento de escoger materiales, planear clases, responder preguntas, y sobre todo en el momento de calificar la producción lingüística de los estudiantes.

Este entrenamiento debe darle al instructor las herramientas necesarias para poder conocer los perfiles estudiantiles basados en conceptos establecidos por la investigación, y no basados en sus creencias personales únicamente. Los instructores de español deben formar parte de una conversación académica que tome en cuenta a los estudiantes de herencia como recursos de instrucción y no como obstáculos en el momento de desarrollar nuevas prácticas metodológicas.

La importancia del español como materia académica a través de las universidades en Estados Unidos es patente. De igual manera, el número de estudiantes que hablan español como lengua de herencia en clases de español en el nivel universitario continuará aumentando al nivel local y nacional. Así, como apuntan Potowski y Carreira (2004), los programas de español en Estados Unidos (y particularmente aquellos al nivel universitario) deben considerar si las presuposiciones y filosofías que moldean sus prácticas pedagógicas y curriculares actuales responden o no a las realidades demográficas del país y de sus estudiantes. Es importante de igual manera que se recluten profesores y estudiantes que entiendan la realidad lingüística y sociopolítica del hablante de herencia del español en Estados Unidos, para así equilibrar la experiencia académica de todos los estudiantes en los departamentos de español a través del país. Más allá de contratar instructores de alguna nacionalidad en particular, el objetivo está perfectamente ilustrado por García-Moya cuando concluye que «la necesidad principal es tener instructores bilingües que demuestren empatía, sea cual sea su origen étnico» (1981: 62).

\section{REFERENCIAS BIBLIOGRÁFICAS}

Carreira, Maria y Olga Kagan (2011): «The Results of the National Heritage Language Survey: Implications For Teaching, Curriculum Design, And Professional Development», Foreign Language Annals, 44, 1, 40-64.

García-Moya, Rodolfo (1981): «Teaching Spanish to Spanish Speakers: Some Considerations For The Preparation Of Teachers», en Valdés, Guadalupe; Anthony G. Lozano y Rodolfo García-Moya, eds., Teaching Spanish to the Hispanic bilingual, New York, Teachers College Press, 59-65.

NORMAS. REVISTA DE ESTUDIOS LINGÜÍITICOS HISPÁNICOS, NÚMERO 3 (AÑO 2013):

http://www.uv.es/normas

(ISSN 2174-7245) 
HoltMeYeR, Dan (2011): «UNL's Hispanic enrollment continues to grow», The Daily Nebraskan (Universidad de Nebraska-Lincoln). En línea: <http://admin.collegepublisher.com/preview/mobile/2.3308/2.3911/1.2621243>.

LYNCH, Andrew (2003). «Toward a Theory of Heritage Language Acquisition», en Roca, Ana y Maria Cecilia Colombi, eds., Mi lengua: Spanish as A Heritage Language in The United States, Washington, D.C, Georgetown University Press, 25-50.

MonTRUL, Silvina (2012): «Is the Heritage Language Like a Second Language?», EUROSLA Yearbook, 12, 1-29.

Pew Hispanic Center (2010): Census 2010 (Nebraska). En línea: <http://www.pewhispanic.org/census-2010/> [consulta: 09/07/2012].

Pew Hispanic Center (2013a): State and county databases. En línea: <http://www.pewhispanic.org/states/county/31079/> [consulta 05/02/2013].

Pew Hispanic Center (2013b): Demographic profile of Hispanics in Nebraska, 2010. En línea: 〈http://www.pewhispanic.org/states/state/ne/> [consulta: 09/02/2013].

Potowski, Kim y María CARReIRA (2004): «Teacher Development and National Standards for Spanish as a Heritage Language», Foreign Language Annals, 37, 3, 427-437.

Potowski, Kim (2005): Fundamentos de la enseñanza del español a hispanohablantes en los EE. UU., Madrid, Arco/Libros.

Potowski, Kim (2008): «¿Por qué ofrecen una clase de español para hispanohablantes?», en Ewald, Jennifer y Anne Edstrom, eds., El español a través de la lingüística: Preguntas y respuestas, Somerville, MA, Cascadilla Press, 228-241.

RocA, Ana (2000): «Introducción», en Roca, Ana, ed., Research on Spanish in the United States: Linguistic Issues and Challenges, Somerville, MA, Cascadilla Press.

U.S. Census Bureau (2011): «The Hispanic Population: 2010 Census Brief». En línea: <http://www.census.gov/prod/cen2010/briefs/c2010br-04.pdf> [consulta: 09/07/2012].

U.S. Census Bureau (2001): «The Hispanic Population: 2000 Census Brief». En línea: <http://www.census.gov/prod/2001pubs/c2kbr01-3.pdf> [consulta: 22/05/2013].

SCHwARTZ, Ana María (2001): «Preparing teachers to work with heritage language learners», en Peyton, Joy Kreeft; Donald Ranard y Scott McGinnis, eds., Heritage Languages in America: Preserving a National Resource, DC/McHenry, IL, Center for Applied Linguistics/Delta Systems, 229-251. 
VALDÉS, Guadalupe (2001): «Heritage Language Students: Profiles And Possibilities» en Peyton, Joy Kreeft; Donald Ranard y Scott McGinnis, eds., Heritage Languages in America: Preserving a National Resource, DC/McHenry, IL, Center for Applied Linguistics/Delta Systems, 37-77.

WELLES, Elizabeth. (2004): «Foreign Language Enrollments in United States Institutions of Higher Education, Fall 2002», ADFL Bulletin, 2-3, 35, 7-26. En línea: <http://www.mla.org/documents\#tab06> [consulta 10/05/2012].

NORMAS. REVISTA DE ESTUDIOS LINGÜÍSTICOS HISPÁNICOS, NÚMERO 3 (AÑO 2013):

http://www.uv.es/normas

(ISSN 2174-7245) 


\section{APÉNDICE. RECURSOS ADICIONALES PARA LA PREPARACIÓN PEDAGÓGICA DE INSTRUCTORES DE ESPAÑOL COMO LENGUA DE HERENCIA}

Hoy en día existe una buena cantidad de recursos de acceso público que ofrecen materiales para la preparación de maestros que tienen estudiantes que hablan español como lengua de herencia al nivel universitario. A continuación se enumeran algunos:

a) El Centro Nacional de Lenguas Extranjeras (NFLC por sus siglas en inglés) ofrece varios módulos pedagógicos para la enseñanza y el aprendizaje del español como lengua de herencia en Estados Unidos (http://www.nflc.org/REACH/).

b) El Centro de Investigación Nacional para las Lenguas de Herencia (NHLRC por sus siglas en inglés) y la Universidad de California en Los Ángeles (UCLA) ofrecen un taller interactivo en línea incluyendo módulos para instructores de lenguas de herencia http://startalk.nhlrc.ucla.edu/default_startalk.aspx).

c) El Centro de Lingüística Aplicada (CAL por sus siglas en inglés) ofrece una lista semi-exhaustiva de los libros de texto más populares y otros recursos útiles de uso para clases de español como lengua de herencia

(http://www.cal.org/resources/archive/rgos/sns- bib.html\#_Toc529686259).

d) El Departamento de Lingüística Hispánica de la Universidad de Illinois en Chicago publica programas de curso de español para hablantes de herencia al igual que otros materiales de interés para la enseñanza del español como lengua de herencia (http://www.uic.edu/orgs/actflsigsns/).

e) El NHLRC mantiene un listserv a través del cual se difunde información pertinente a la enseñanza de lenguas de herencia en Estados Unidos (para suscribirse, se debe enviar un mensaje a heritage-on@lists.nflc.org).

f) La Iniciativa para las Lenguas de Herencia, un proyecto dirigido por el Centro de Lingüística Aplicada y el Centro Nacional de Lenguas Extranjeras, provee información, materiales y oportunidades profesionales para aquellos interesados en la enseñanza de las lenguas de herencia en Estados Unidos (http://www.cal.org/heritage/).

NORMAS. REVISTA DE ESTUDIOS LINGÜÍSTICOS HISPÁNICOS, NÚMERO 3 (AÑO 2013):

http://www.uv.es/normas

(ISSN 2174-7245) 
